\title{
"Prevalence of urinary tract infections and their antibiotic sensitivity in tertiary care hospital Lahore"
}

\author{
Ahmad Naeem Sajed ${ }^{1}$, Uzma Batool ${ }^{3}$, Dr. Shaghufta Iram² ${ }^{2}$, Prof. Dr. Noshin \\ Wasim Yousaf ${ }^{2}$, Muhammad Nadeem Asghar ${ }^{4}$, Sana Khan ${ }^{2}$, Muhammad Naeem \\ Mukhtar ${ }^{4}$, Mubashir $\mathrm{Akbar}^{4}$, Sajid $\mathrm{Ali}^{4}$ \\ ${ }^{I}$ Institute of Molecular Biology and Biotechnology The University of Lahore-Pakistan \\ ${ }^{2}$ Pathology Department Allama Iqbal Medical College Lahore-Pakistan \\ ${ }^{3}$ National University of Science and Technology Islamabad-Pakistan \\ ${ }^{4}$ Emergency Services Academy Rescue 1122 Lahore-Pakistan
}

\begin{abstract}
This cross sectional study was conducted to determine the prevalence of UTI among population visiting Jinnah Hospital Lahore to identify microorganisms responsible for UTI to explore sensitivity patterns of identified microorganisms to certain antibiotics used in the cure of UTI. This study included 378 samples of all age groups. In this study we utilized two main approaches, questionnaire and urine testing (urinalysis and urine culture). 151 samples showed no growth on culture plates for taking antibiotics at the time of collection of samples. The positive samples for pathogenic microorganisms were 197. Out of those positive cases gram negative bacteria were found responsible for $81.92 \%$ of UTIs in comparison to gram positive bacteria which were $18.08 \%$. Escherichia coli were the most predominant uro-pathogen with $34 \%$ followed by Klebsiella spp. $18.78 \%$ and Staphylococcus spp. $12.67 \%$. The prevalence of Candida spp. $20.30 \%$ was also very high in males and females. Urine culture is an important diagnostic tool to confirm UTI. Rules must be put on the consumption of antibiotics to limit their abuse and misuse. Physicians are advised to use antibiotics like sulzone, nitrofuration, tazocin and imipenum for gram negative bacteria and tetracycline against gram positive bacteria, these antibiotics showed a low resistance rate in that study over commonly used antibiotics.
\end{abstract}

Key words: Urinary tract infections, pathogenic microorganisms, susceptibility.

\section{Introduction}

Urinary tract infection (UTI) is a term applied to a variety of clinical conditions ranging from asymptomatic presence of bacteria in the urine to rigorous infection of the kidney with resultant sepsis ${ }^{1}$. Urinary tract infections (UTIs) are major public health problem affecting millions of peoples and are the second most common type of infections in the body. Universaly about 150 million people are diagnosed with UTI each year ${ }^{2}$. These are the most common bacterial infections encountered by clinicians in developing countries. UTIs can occur at any time in the life of an individual. Urinary tract infections occur in all age groups including men and women ${ }^{3}$.

Clinical symptoms of UTI usually include wanting to urinate more often and urgently, burning pain or a scalding sensation during urination, urine that appear bright pink or cola color and a sign of blood in the urine. A feeling that the bladder is still full after passing urine, cloudy strong smell in urine, pain above the pubic bone, rectal pain in men, pelvic pain in women and tenderness with pyuria ${ }^{4}$. In young children, the only symptom of a urinary tract infection may be a fever. Infants may feed poorly, vomit, sleep more or show signs of jaundice. In older children, new onset urinary incontinence (loss of bladder control) may occur ${ }^{5}$.

The normal microbial floras are those organisms that make their home on or in some part of the body. The normal flora is also found in vagina and urethra. In healthy person such microorganisms rarely cause disease. After birth, the peri-urethral area becomes colonized with normal microbial flora. These organisms act as a defense barrier against UTI pathogens. The normal flora may destroy when respiratory tract infections are treated with broad spectrum antibiotics. The primary normal microbial floras of a healthy person are of the genus lactobacillus such as Lactobacillus acidophilus and Lactobacillus doderlein. They produce lactic acid (some species produce hydrogen peroxide or antibiotic) in combination with fluids secreted during sexual arousal and are to a great extent responsible for the characteristic odor associated with the vaginal region. During the period of menstruation vaginal microbial flora concentration decreases leading high risk of UTIs ${ }^{6}$.

Approximately $95 \%$ of cases of UTIs are caused by bacteria that normally multiply at the opening of the urethra and move up to the bladder. Most infections are caused by retrograde ascent of bacteria from the fecal flora through urethra to the bladder and kidney particularly in the females who have a shorter and wider urethra than males. Approximately all UTIs are ascending in origin and are caused by bacteria in the gastro intestinal tract that have occupied the peri-urethral $\mathrm{area}^{7}$. The higher prevalence in females as compared with 
males is more accountable to contamination during sexual intercourse, urethra massage and even urination with chronic pathogens that resides in the perennial skin. In males the organisms often originate from the sub pepucial sac. Men with prostate problems such as an inflamed prostate gland that can cause the bladder to only partly empty, babies especially those born with physical problems (congenital abnormalities) of the urinary system are susceptible to UTIs ${ }^{8}$.

It is very necessary to identify organisms causing urinary tract infections and treat them as soon as possible to avoid any long term complications and to reduce the risk of any significant morbidity and suspicion. Understanding the prevalence of urinary tract infections in different populations will help to guide the appropriate level of suspicion and the appropriate work-up for urinary tract infection ${ }^{9}$. Treatment of urinary tract infections requires understanding of the pathogenesis of UTI and the role of host and bacterial factors. Only with insight into this process, we can get better our ability to spot patients at risk. Careful diagnosis and treatment results in successful resolution of infections in most instances, only with insight into this process we can enhance our ability to identify patients at risk ${ }^{10}$.

\section{Material And Methods}

This study was carried out on the patients visiting Jinnah hospital Lahore and conducted in Microbiology laboratory department of Allama Iqbal Medical College Lahore. Over the course of study, total of 378 urine samples were collected. Out of those 378 successful samples 194 females and 184 males and were in the age group of 1 to above 60 years old. There were higher numbers of samples collected from the extreme of ages $1-9$ years $(21.739 \%)$ and above 60 years $(22.826 \%)$.

Table 1 Showing age and gender wise distribution of study participants

\begin{tabular}{|c|c|c|c|c|c|c|}
\hline \multirow{2}{*}{ Age groups (years) } & \multicolumn{2}{|c|}{ Males } & \multicolumn{2}{|c|}{ Females } & \multicolumn{2}{|c|}{ Total } \\
\hline & No. & (Percentage) & No. & (Percentage) & No. & (Percentage) \\
\hline $1-9$ & 40 & $(21.739)$ & 32 & $(16.495)$ & 72 & $(19.04)$ \\
\hline $10-19$ & 15 & $(08.153)$ & 30 & (15.463) & 45 & $(11.90)$ \\
\hline $20-29$ & 31 & $(16.847)$ & 35 & (18.042) & 66 & $(17.46)$ \\
\hline $30-39$ & 22 & $(11.956)$ & 36 & $(18.556)$ & 58 & $(15.34)$ \\
\hline $40-49$ & 19 & $(10.326)$ & 19 & (09.793) & 38 & $(10.05)$ \\
\hline $50-59$ & 15 & $(08.153)$ & 13 & (06.702) & 28 & $(07.40)$ \\
\hline$>60$ & 42 & $(22.826)$ & 29 & $(14.949)$ & 71 & $(18.78)$ \\
\hline Total & 184 & $(100)$ & 194 & $(100)$ & 378 & $(100)$ \\
\hline
\end{tabular}

\subsection{Collection of urine specimen}

The specimens were collected into sterilized, wide necked, leak proof, plastic universal containers. Clean-voided mid-stream urine specimens were collected. Each of the study participant was instructed on the mode of collection of the mid-stream urine that was during forceful urination after the first $10-20 \mathrm{ml}$ has been voided $^{11}$.

\subsection{Sample Preparation and Microscopy}

The collected urine sample was prepared for microscopy according to standard method. The urine samples were mixed and aliquots centrifuged at $5000 \mathrm{rpm}$ for $5 \mathrm{~min}$. The deposits were examined using both $10 \mathrm{X}$ and $40 \mathrm{X}$ objectives. Samples with $\geq 6$ white blood cells $/ \mathrm{mm}$ were regarded as pyuric ${ }^{12}$.

\subsection{Media preparation}

The media used were Nutrient Agar (NA) and Cysteine Lactose Electrolyte Deficient (CLED) Agar as described by Cheesbrough, 2005. Other media were prepared according to the manufacturer's instructions.

\subsection{Sample culturing}

The collected urine samples were processed for culture and report according to standard method procedure and protocol. For the visual examination and calibrated Loop Streak method for urine culture performed $1 \mu \mathrm{l}$ wire loop was sterilized and dipped of into urine sample. The loop was then streaked on the plate of Cysteine Lactose Electrolyte Deficient (CLED) Agar. The plates were incubated over night at $37^{\circ} \mathrm{C}$ for 24 to 48 hours. Following the appearance growth well-isolated bacterial colonies were processed for antibiotic sensitivity and biochemical tests ${ }^{13}$.

\subsection{Identification of microorganisms}

All bacterial isolates were characterized on the basis of colony morphology, cellular morphology, Gram staining, Catalase test, D Nase and oxidase tests. From the results of preliminary identification test the 
gram negative rods were subjected to the following routine biochemical tests such as motility test, indole production, urease production, citrate utilization test and Triple sugar iron reaction ${ }^{14}$.

\subsection{Sensitivity test}

All isolated organisms were tested against various antibiotics in vitro, antibiotic susceptibility was tested in laboratory by disc diffusion assay on Muller Hinton Agar by modified Kirby-Bauer method ${ }^{15}$ using the following antibiotic discs, Augmentin, Penicillin, Sulzone, Cefotaxime, Cefoperazone, Amikacin, Gentamicin, Ciprofloxacin, Nalidixic acid, Imipenum, Novobiocin, Tazocin, Erythromycin, Tetracycline and Nitrofuration.

\subsection{Statistical analysis}

Data was tabulated and analyzed using the Statistical Package for Social Sciences SPSS 16 software. Data was presented as frequencies. Evaluations were carried out at $95 \%$ confidence level and $\mathrm{P}<0.05$ was considered statistically significant.

\section{Results And Discussion}

\begin{tabular}{|c|c|c|c|c|c|c|c|c|}
\hline \multirow{2}{*}{$\begin{array}{c}\text { Age } \\
\text { groups } \\
\text { (years) }\end{array}$} & \multicolumn{2}{|c|}{ Positive samples of Males } & \multicolumn{2}{|c|}{ Negative samples of Males } & \multicolumn{2}{|c|}{ Positive samples of Females } & \multicolumn{2}{|c|}{ Negative samples of Females } \\
\hline & & (\% age) & & (\% age) & & (\% age) & No. & (\% age) \\
\hline $1-9$ & 16 & (16) & 24 & $(28.57)$ & 08 & $(08.24)$ & 24 & $(24.74)$ \\
\hline $10-19$ & 09 & (09) & 06 & $(07.14)$ & 18 & $(18.55)$ & 12 & (12.37) \\
\hline $20-29$ & 17 & (17) & 14 & (16.67) & 20 & $(20.62)$ & 15 & (15.46) \\
\hline $30-39$ & 15 & (15) & 07 & $(08.34)$ & 13 & $(13.41)$ & 23 & $(23.71)$ \\
\hline $40-49$ & 13 & (13) & 06 & $(07.14)$ & 13 & $(13.41)$ & 06 & $(06.19)$ \\
\hline $50-59$ & 09 & (09) & 06 & (07.14) & 08 & $(08.24)$ & 05 & $(05.16)$ \\
\hline$>60$ & 21 & (21) & 21 & $(25.00)$ & 17 & $(17.53)$ & 12 & (12.37) \\
\hline Total & 100 & $(100)$ & 84 & $(100)$ & 97 & $(100)$ & 97 & $(100)$ \\
\hline
\end{tabular}

Table 2 Showing age and gender wise distribution of positive and negative samples of study participants

Out of total collected 378 successful samples 194 females and 184 males and were in the age group of 1 to above 60 years old. There were higher numbers of samples collected from the extreme of ages 1-9 years (21.739\%) and above 60 years (22.826\%). In age groups of 1-9 years and above 60 years there were higher numbers of negative samples of males and females showing no growth on culture plates. That could be due to indiscriminate consumption of antibiotics by the peoples since some of them confessed using non prescribed antibiotics whenever difficulty in urination was noticed. UTIs were higher in numbers of males than females in age group of 1-9 years because of continuous wetting and due to difficulty in keeping dry under wears. The male positive samples above 60 years old showed high risk of infections than females due to bladder outlet obstruction in older men there might be bacterial prostatitis and non bacterial prostatitis. For the age group 30-39 years again males showed higher risk of infections than females because younger men represented complication of sexually transmitted urethritis. However, in 10-19 year's and 20-29 age groups female's samples were high as compared to the males because of shortness of urethra which make easy excess of pathogens in female urinary tract. Finally for the age groups 40-59 there was a slight difference of positive and negative samples in males and females. Both the extremes of age groups have risk of UTIs infections.

Table 3 Showing percentage of isolation of microbes from UTIs

\begin{tabular}{|c|c|c|c|c|c|c|}
\hline Organisms Isolated & Males & \%age & Females & \%age & Total & \%age \\
\hline E. coli & 37 & (37) & 30 & $(30.93)$ & 67 & $(34.01)$ \\
\hline Klebsiella & 22 & (22) & 15 & $(15.46)$ & 37 & $(18.78)$ \\
\hline S. aureus & 05 & $(05)$ & 08 & $(08.25)$ & 13 & $(06.60)$ \\
\hline S. epidermydous & 05 & $(05)$ & 04 & $(04.12)$ & 09 & $(04.57)$ \\
\hline S. saprophyticus & 01 & $(01)$ & 02 & $(02.06)$ & 03 & $(01.52)$ \\
\hline Pseudomonas & 05 & $(05)$ & 04 & $(04.12)$ & 09 & $(04.57)$ \\
\hline Proteus & 01 & $(01)$ & 02 & $(02.06)$ & 03 & $(01.52)$ \\
\hline H. Streptococci & 04 & $(04)$ & 07 & $(07.22)$ & 11 & $(05.58)$ \\
\hline Neisseria gonorrhea & 02 & $(02)$ & 00 & $(00.00)$ & 02 & $(01.02)$ \\
\hline Citrobector & 01 & $(01)$ & 02 & $(02.06)$ & 03 & $(01.52)$ \\
\hline Candida & 17 & $(17)$ & 23 & $(23.72)$ & 40 & $(20.31)$ \\
\hline Total & 100 & $(100)$ & 97 & $(100)$ & 197 & (100) \\
\hline
\end{tabular}

Out of total 378 collected samples 181 samples showed no growth on culture plates. 197 were positive samples. Pathological microbes were isolated from 197 positive samples $(52.11 \%)$ which in the vast majority of 
cases E. coli $(34.01 \%)$ and Klebsiella species $(18.78 \%)$ were among the most frequent uropathogen bacteria. That was similar to other studies where it was the most frequent pathogen causing UTI as studied by Mehar where $62.6 \%$ cultures grew $E$. coli $^{16}$ and $66 \%$ E. coli studied by Naeem in Islamabad ${ }^{17}$. Those results were also similar with a study conducted by Dilnawaz in 2005 in India which reflects that first two common organisms were E. coli and Klebsiella pneumonae ${ }^{18}$; both microorganisms were different with respect to individual percentage from previous studies. The prevalence of the UTI isolates in relation to gender was shown in the Table 3, E. coli $(34.01 \%)$ was the most frequently isolated pathogen in both sexes while prevalence of Klebsiella was higher in males than females. More isolates of Staphylococcus species and H. Streptococci were recovered more from females than males. Two cases of Neisseria gonorrhea were positive in males. Candida was most prevalent after $E$. coli in both sexes but percentage was higher in females. There was no prominent sex difference for the remaining isolates i.e. Proteus, Pseudomonas and Citrobector. Most of the cases of UTIs were due to the gram negative bacilli 81.93 percent. Gram negative bacilli cases were 9.55-\% higher in males than females. However the gram positive cocci were less prevalent than gram negative bacilli. The prevalence of gram positive cocci was higher in female than males. Consequently higher prevalence of $E$. coli $(34.01 \%)$ and Klebsiella spp. (18.78\%) might be due to fecal contamination, the predilection of the organisms from the toilets and the shortness of the female urethra in females. That prevalence however was also reported in earlier works by Smith in Australia ${ }^{12}$.

There was also a possible link between the prevalence of UTI among population and the level of personal hygiene or the state of toilet facilities. Most of the peoples examined rated the toilets as bad. Bad in that context implies that there was no sufficient provision of water to dirt free and wash out the toilets on public places congestive areas frequently. When unclean there was an accumulation of urine sediments forming a bulky scum. In that case population could become contaminated during urination, that was observed by visiting different areas in Lahore and through by asking questions from population. Sexual activity was another feature that predisposes population to UTI. For example Staphylococcus aureus (6.60\%) which was a member of skin flora might stay on the skin and get transmitted during sexual intercourse. On examination of demographic state of the cases by age, it was observed that both the extremes of age groups have risk of UTIs. There were many cases of UTIs caused by candida spp. (20.30\%). Candida urinary infection was usually found in those with immune-suppressant ${ }^{12}$. The prevalence of candida was high in this study as compared to previous studies.

\begin{tabular}{|c|c|c|c|c|c|c|c|c|c|c|c|}
\hline \multirow[t]{2}{*}{ Organisms } & \multicolumn{11}{|c|}{ Antibiotics } \\
\hline & AMC & $\mathrm{AK}$ & CIP & CTX & $\mathrm{CPZ}$ & GEN & IMP & NA & NIT & SCF & TZP \\
\hline E. coli & 18.7 & 53.1 & 26.0 & 70.0 & 13.3 & 92.30 & 90.6 & 30.78 & 74.3 & 78.0 & 72.7 \\
\hline Klebsiella & 16.6 & 61.5 & 18.8 & 69.3 & 33.3 & 15.4 & 93.3 & 25.0 & 58.33 & 52.94 & 60.0 \\
\hline Pseudomonas & 51.5 & 87.1 & 1.00 & 69.1 & 73.6 & 66.7 & 60.0 & 8.0 & 20.0 & 71.4 & 83.3 \\
\hline Proteus & 68.0 & 72.0 & 25.0 & 99.0 & 80.6 & 50.0 & 70.6 & 67.4 & 66.7 & 66.7 & 88.5 \\
\hline Citrobecteria & 71.2 & 53.3 & 21.0 & 46.0 & 48.0 & 63.8 & 70.0 & 28.0 & 87.8 & 72.0 & 50 \\
\hline N. gonorrhea & 64.4 & 22.6 & 16.0 & 11.1 & 14.5 & 18.5 & 77.7 & 19.6 & 21.1 & 61.2 & 45.7 \\
\hline
\end{tabular}

Table 4 Percentage susceptibility of Gram negative isolates of UTIs

AMC - Augmentin, AK -Amikacin, CIP - Ciprofloxacin, CTX - Cefotaxime, CPZ - Cefoperazone, GEN Gentamicin, IMP - Imipenum, NA - Nalidixic acid, NIT - Nitrofuration, SCF - Sulzone, TZP - Tazocin

In this study, it was observed that the susceptibility of the isolates to the tested antibiotics differed with the species. Of the E. coli isolates (34.01\%) showed resistance to the commonly used antibiotics. The highest sensitivity was shown by gentamicin $(92.30 \%)$, imipenum (90.6\%), sulzone (78\%), tazocin (72.7 \%), cefotaxime (70\%) and nitrofuration (74\%). Of the klebsiella isolates only sensitive to imipenum (93.3\%). Studied by Uwaezuoke in Nigeria in 2006, nitrofurantoin and gentamicin were found to be $80 \%$ effective against strains of $E$. coli $^{19}$. The sensitivity was almost same in that study as studied by Uwaezuoke. klebsiella isolates were highly resistant to all tested antibiotics. All isolate of gram negative bacteria were not commonly sensitive to all tested antibiotics.

Table 5 Percentage susceptibility of Gram positive isolates of UTIs

\begin{tabular}{|c|c|c|c|c|c|c|c|c|c|c|c|}
\hline \multirow[b]{2}{*}{ Organisms } & \multicolumn{11}{|c|}{ Antibiotics } \\
\hline & AMC & CIP & CTX & $\mathrm{CPZ}$ & $\mathrm{E}$ & GEN & NA & NIT & $\mathrm{P}$ & TE & $\mathrm{NV}$ \\
\hline S. aureus & 66.7 & 72.7 & 60.0 & 56.7 & 42.9 & 77.8 & 40.0 & 66.7 & 25.0 & 72.7 & 63.3 \\
\hline S. epidermydous & 20 & 16.66 & 56.1 & 48.8 & 50.0 & 20.3 & 41.5 & 80.0 & 15.0 & 62.5 & 100 \\
\hline S. saprophyticus & 40.0 & 33.3 & 70.1 & 60.0 & 33.3 & 40 & 50.0 & 68.9 & 40 & 66.7 & 00.0 \\
\hline H. streptococci & 33.3 & 14.2 & 10.4 & NA & 50.0 & 10.0 & 11.3 & 75 & 00.0 & 97.0 & 98.0 \\
\hline
\end{tabular}

AMC - Augmentin, CIP - Ciprofloxacin, CTX - Cefotaxime, CPZ - Cefoperazone, E - erythromycin, GEN Gentamicin, NA- Nalidixic acid, NIT - Nitrofuration, P - penicillin, TE - tetracycline, NV- novobiocin. 
Staphylococcus aureus was among most frequently isolates which was highly susceptible to gentamicin (77.8\%), ciprofloxacin $(72.7 \%)$ and tetracycline $(72.7 \%)$. Staphylococcus epidermydous was $100 \%$ sensitive to novobiocin and highly susceptible to nitrofuration (80.0\%). Staphylococcus saprophyticus was sensitive to cefotaxime (70\%). It was $100 \%$ resistant to novobiocin. Haemolytic streptococci showed high susceptibility to tetracycline (97.0\%). nitrofuration (75\%) and novobiocin (98\%). There was no commonly tested antibiotic for isolated Gram positive bacteria.

A particularly remarkable result was obtained for gram negative bacteria with cefotaxime, sulzone, nitrofuration, tazocin and imipenum. Tetracycline and novobiocin were commonly and highly effective antibiotics for isolated gram positive bacteria. Those antibiotics are still effective for treatment of urinary tract infections. The majority of antibiotics tested according to table 4 and 5 resistance of uropathogens against majority of commonly used antibiotics increased. The implication of that was the possibility of easy access causing self-medication, misuse and abuse, leading to the development of resistance. The selection of antibiotic against any urinary tract pathogen depends on the antibiotic resistance pattern, its pharmacokinetic properties, dose quantity and timings, its effect on gastrointestinal tract and vaginal flora allergies and adverse effects caused by that drug ${ }^{20}$. Antimicrobial resistance was a big problem cause of great concern throughout the world. Knowledge of the antibacterial resistance among uropathogens was essential to provide appropriate cost effective therapy.

\section{Conclusion}

Community must be well-informed on how to make use of the toilets by themselves in a secure and hygienic way; urine culture is a significant diagnostic tool to authenticate UTI. Empirical antibiotic choice in cure of UTI should be based on the knowledge of local prevalence of causative micro-organisms and their antimicrobial sensitivities rather than on universal guidelines. Unsystematic recommendation and use of antibiotics should be disheartened in both the public and hospital setting by incessant public awareness on rational antibiotics use as well as taking on stern national antibiotic policy to standardize the recommendation, sale and use of antibiotics.

\section{Acknowledgements}

I am very thankful to Institute of Molecular Biology and Biotechnology The University of Lahore and Pathology Department Allama Iqbal Medical College Lahore-Pakistan.

\section{References}

[1]. Tanagho, Emil A, Mcaninch, Jack W. Smith's General Urology: United States of America. McGraw-Hill companies Inc; Bacterial Infections of the genitourinary tract, 2004, 203-227.

[2]. Owa JA. Urinary tract infections in children, Peadiatrics and child health in tropical region, 2(2), 2007.

[3]. Tessema B, Kassu A, Mulu A, Yismaw G. Predominant isolates of urinary tract pathogens and their antimicrobial susceptibility patterns in Gondar University Teaching Hospital North West Ethiopia. Ethiop. Med. J, (1), 2007, 61-67.

[4]. Car J. Urinary tract infections in women: diagnosis and management in primary care. BMJ, (332), 2006, 94-97.

[5]. Bhat RG, Katy, TA, Place FC. Pediatric urinary tract infections. Emergency medicine clinics of North America., 29 (3), 2011, 637653.

[6]. Vasquez A, Jakobsson T, Ahrne S, Forsum U, Molin G. Vaginal Lactobacillus Flora of Healthy Swedish Women. J. of Clin. Micro, 40 (8), 2002, 2746-2749.

[7]. John ED and Michel L. Urinary tract infections during pregnancy. J. Am. Fam. Physiian., 61(3), 2006, 713-720.

[8]. Starr C, Taggart RC. Biology the unit and diversity of life. Wards worth publishing Co. Belmouth co, (3), 2002, 509-533.

[9]. Quigley. Diagnosis of Urinary Tract Infections in children. Curr. Opin. Pediatr, 21(2), 2009, 124-128.

[10]. Masson P, Matheson S, Webster AC, Craig J C. Meta-analyses in prevention and treatment of urinary tract infections. Infect. Dis. Clin. North Am, (23), 2009, 355-385.

[11]. Forbes BA, Sahm DF, Weissfeld AS. Bailey and Scott's Diagnostic microbiology, Mosby Elsevier, (12), $2007,842-855$.

[12]. Smith PJ, Morris AJ, Reller LB. Predicting Urine Culture Results by Dipstick Testing and Phase Contrast Microscopy. Pathol, 35 (2), 2003, 161.

[13]. Cheesbrough Moneca. District Laboratory Practice in Tropical Countries, 2 (2), 2005, 105-114.

[14]. Fawole E, Oso. An introduction to laboratory Manual of Microbiology. University printing press Ibadan Nigeria, (31), $1998,23-34$.

[15]. WHO SEARO, Guidelines on standard operating procedures for Microbiology- antimicrobial susceptibility testing. 2006.

[16]. Mehar TM, Khan H, Mohammad Khan T, Iqbal S, Adnan S. E. coli urine superbug and its antibiotic sensitivity. J. Med. Sci., (8), 2010, 110-113.

[17]. Naeem M, Khan M, Qazi SM.,. Antibiotic susceptibility pattern of bacterial pathogens causing urinary tract infection in a tertiary care hospital. Ann. Pak. Inst. Med. Sci, (6), 2010, 214-218.

[18]. Sheikh D, Ashfaq S, Sheikh K and Sheikh M. Studies on resistancel sensitivity pattern of bacterial related with urinary tract infections. Med. J. Isl. World Acad. Sci., (15), 2005; 129-133.

[19]. Uwaezuoke JC, Ogbulie JN. Antibiotic sensitivity pattern of urinary tract pathogens in port - Harcourt, Nigeria. J. appl. Sci. environ Mgt., (10), 2006, 103-107.

[20]. Zareef S, Zafar H, Izhar K, Dodhy, Ehsan M, Hayat A. The culture and sensitivity pattern of uropathogens detected at Benazir Bhutto hospital. Ann. Pak. Inst. Med. Sci., (5), 2009, 121-12. 